\title{
Rhythm Disorders - Heart Beat Classification of an Electrocardiogram Signal
}

\author{
Faiza Iftikhar \\ Lab. Engineer \\ Department of Electrical Engi- \\ neering \\ Lahore College for Women \\ University, Lahore, Pakistan
}

\author{
Ayesha Shams \\ Research Scholar \\ Department of Electrical Engi- \\ neering \\ University of Engineering and \\ Technology, Lahore
}

\author{
Arfa Dilawari \\ Research Assistant \\ Alkhawarizmi Institute of Com- \\ puter Science \\ University of Engineering and \\ Technology, Lahore
}

\begin{abstract}
Arrhythmia disorders play a vital role in heart diseases progression. Detection and treatment of arrhythmia disorders can help indirectly in controlling the heart disease. In hospitals, physicians classify the beats after examining the electrocardiogram (ECG) report. Sometimes, physicians are not that expert to diagnose the arrhythmias correctly and accurately. In these circumstances, there is a need for automatic and accurate heart beat classifier which takes the ECG signal as an input and classify it into different rhythm disorders. In this paper, an arrhythmia disorder classifier is designed and developed using Feedforward Backpropagation neural network. The supervised network is trained based on the features extracted from the ECG databases of MIT-BIH. The trained network will classify the beats into premature atrial/ventricular contraction (PAC/PVC), left/right bundle branch block (LBBB/RBBB), paced beat and normal beat. This automatic system will make the treatment faster even in the absence of expert physicians.
\end{abstract}

\section{General Terms}

ECG, Rhythm disorder, Neural Network, Feed Forward, Peaks P, Q, R, S, T, Classifier, Detection.

\section{Keywords}

QRS Complex, Mean Power Frequency, Power Spectral Density, Fiducial Point, LBBB, RBBB, PVC, APC, Paced, purelin, logsig.

\section{INTRODUCTION}

The electrocardiogram is very helpful in the diagnosis of cardiac diseases since they often result in ECG abnormalities. To detect such abnormalities, a variety of methods have been used in the literature. In this section, we will review the characteristics of ECG and rhythm disorders to determine the features for training of feed forward neural network.

\subsection{The Electrocardiogram (ECG)}

The heart is the most vital organ of the human body since it acts as a pump that pushes oxygen rich blood to the organs, cells, and tissues of the body. Every heart beat is caused by electrical impulses from the heart muscle that causes the atria and then the ventricles to contract and consequently pump blood to the lungs and the rest of the body. This electrical activity of the heart is measured by the electrocardiogram which serves as a means to detect for irregular heart conditions and possibly heart diseases [1]. Electrocardiography has evolved over time and is becoming more accurate as it is being automated by using software.
The electrical signal that stimulates the heart beat starts from the sinoatrial node (SA) which is known as the heart's "natural pacemaker" and is located at the top of the right chamber/atrium. This signal branches through atria's, causing them to contract and pump blood to the lower chambers, the ventricles, where the signal continues via the atrioventricular node (AV). The heart may beat abnormally, if there is pacemaker interruptions which will affect the blood circulation through the patient's body. ECG is the recording of electrical pulses and every heart beat is displayed as a sequence of electric waves distinguished by peaks and valleys. Therefore it is important to record the ECG signal since it allows for the detection of abnormalities or diseases in the heart. The recording of the ECG usually takes place by placing electrodes to measure potentials on the surface of specific body parts usually the arms, legs and the chest. There are three basic techniques used in clinical electrocardiography. The most common is the standard clinical electrocardiogram that utilizes different potential differences called ECG leads. The standard 12 lead ECG is based on 3 limb leads (I, II, III), 3 augmented leads (limb potential relative to a modified Wilson terminal, $\mathrm{aVR}, \mathrm{aVL}, \mathrm{aVF}$ ) and 6 leads placed across the front of the chest and referenced to the Wilson terminal (Lead V1, V2, V3, V4, V5, V6) [2].

\subsubsection{The ECG Characteristics}

An ECG gives two major kinds of information. First, by measuring time intervals on the ECG, the duration of the electrical wave crossing the heart can be determined and consequently we can determine whether the electrical activity is normal or slow, fast or irregular. Second, by measuring the amount of electrical activity passing through the heart muscle, a pediatric cardiologist may be able to find out if parts of the heart are too large or are overworked. The range for frequency and amplitude in volts of an ECG signal is $0.05 \mathrm{~Hz}$ to $100 \mathrm{~Hz}$ and $1 \mathrm{mV}$ to $10 \mathrm{mV}$ respectively. The ECG signal is qualified by five peaks labeled as $\mathrm{P}, \mathrm{Q}, \mathrm{R}, \mathrm{S}$ and T. The reliable and accurate detection of above peaks and QRS complex marks the good performance of ECG analysis system. The upper chamber activation of heart is represented by $\mathrm{P}$ wave and excitation of lower chamber is heart is represented by $\mathrm{T}$ wave. The most important task in ECG signal analysis is QRS complex detection. Once the QRS complex is marked, the other features like heart rate, ST segment, etc., are collected. 


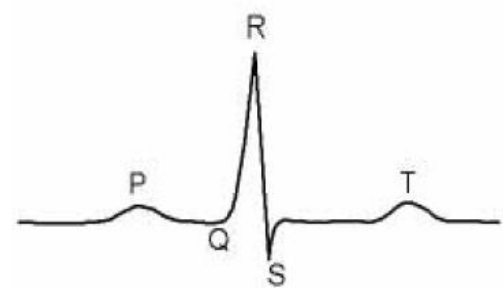

Fig 1: The ECG Signal and its Different Components

In the normal state of heart, PR segment duration ranges from 120 to $200 \mathrm{~ms}$ and QRS interval from 40 to $120 \mathrm{~ms}$ and QT segment duration is less than $420 \mathrm{~ms}$. The normal heart rate is 60 to 100 beats per minute [3]. The shape of ECG signal is the best tool for accessing patient's heart activity.

Table 1: Normal state of the Heart

\begin{tabular}{|c|c|c|}
\hline Parameter & $\begin{array}{c}\text { Normal } \\
\text { Range }\end{array}$ & Unit \\
\hline Heart Rate & $60-100$ & Beats per minute \\
\hline PR Interval & $0.12-0.20$ & $\mathrm{~S}$ \\
\hline QT Interval & $0: 39-0: 04$ & $\mathrm{~S}$ \\
\hline P wave duration & $<0.12$ & $\mathrm{~S}$ \\
\hline QRS width & $0.06-0.1$ & $\mathrm{~S}$ \\
\hline
\end{tabular}

\subsubsection{Einthoven's Triangle}

Willem Einthoven opens up a new area of thought through the practice of ECG in late 1800's. he pioneered a system that was able to measure the potential difference between different points on the body. The electrical activity of the heart is the result of plotted potential values across time. In order to process this, the patient would place a limb in a jar filled with salted water then the patient would place another limb into a separate jar filled with salted water and the potential difference is estimated with the help of a machine connected with both the jars. Einthoven has experimented with different combinations of limbs and he found that different limb combinations gave different results. Each result gave a different view of heart electric activity. He labeled each combination with a lead and observed that some leads have better electrical activity results than other. After much experimentation, Einthoven developed three standard "limb leads" [4].

Todays, we use small electrodes at the place of salted water jars for ECG recording from patient's body. The simplest ECG device is able to record at three leads. These three leads are named as limb leads. Limb lead II is used most frequently. It is the view heart activity from the right arm to left leg [3].

Lead I Right arm Left arm

Lead II Right arm Left leg

Lead III Left arm Left leg

\subsection{Arrhythmia / Rhythm Disorders}

An arrhythmia is a change in the regular beat of the heart. The heart consists of four chambers: two atria located on the top and two ventricles located on the bottom. Arrhythmias may result in either slow or fast heart rates. Additionally, arrhythmias may be either regular or irregular [5]. Sometimes, the heart looks like skipping a beat or the beat appears to be abnormal in an arrhythmia diagnosis and apparently there is no proper reason for such abnormality. Some of the reasons for irregular heart rhythm are cough, tobacco, cold medicines, alcohol and diet pills. The people having rhythm disorders and severe symptoms of illness required to be treated to keep heart beat normal. With rhythmic disorders the patient may feel that his heart is beating at a faster pace can also experience pain in the chest and may notice irregularities or missing heartbeat. With the rhythm disorder, the patient may also experience dizziness and breathlessness [4]. Two basic types of arrhythmia exist: those types originating in the atria and those originating in the ventricles. We are working on some of the very common types of arrhythmias in our research, which are as follows:

\subsubsection{Left Bundle Branch Block (LBBB)}

LBBB is a cardiac conduction abnormality seen on the electrocardiogram (ECG). In this condition, activation of the left ventricle is delayed, which results in the left ventricle contracting later than the right ventricle. The criteria to diagnose a left bundle branch block on the electrocardiogram:

- The heart rhythm must be supraventricular in origin

- The QRS duration must be $=$ or $>120 \mathrm{~ms}$

- There should be a QS or rS complex in lead V1

- There should be a monophasic $\mathrm{R}$ wave in leads I and V6.

The deflection of T wave should be opposite to deflection of QRS complex. This is called $\mathrm{T}$ wave consonance with bundle branch block. This form of $\mathrm{T}$ wave indicates suggest ischemia or myocardial infarction [6].

\subsubsection{Right Bundle Branch Block (RBBB)}

Right Bundle Branch Block (RBBB) is the imperfection of electric conduction system of heart. The right ventricle is not excited directly by electric impulses moving through the right bundle branch (RBB) in case of right bundle branch block, whereas the left bundle branch excites the left ventricle. The electric impulses move through left ventricle's myocardium to the right ventricle and activate the right ventricle [6]. The criteria to diagnose a right bundle branch block on the electrocardiogram:

- The heart rhythm must be supraventricular in origin

- $\quad$ The QRS duration must be $=$ or $>120 \mathrm{~ms}$

- There should be a terminal R wave in lead V1 (e.g., $\mathrm{R}, \mathrm{rR}$ ', rsR', rSR' or qR')

- $\quad$ There should be a slurred S wave in leads I and V6.

\subsubsection{Premature Ventricular Contraction $(P V C)$}

This is also named as ventricular premature beat (VPB) or extrasystole. Extrasystole is a form of abnormal heart beat in which ventricles contract prematurely. This is also taken as palpitations. The ventricle activates the depolarization of cardiac mycytes rather than sinoatrial node. Premature ventricle contractions impart heart rate anomalies. The properties of this turbulence are used to evaluate cardiac functions [7]. 


\subsubsection{Premature Atrial Contractions (PACs)}

The rhythm disorder started in the upper two chambers of heart is called premature atrial contraction (PAC). This a type of irregular heart beat and it is not as serious as premature ventricle contraction (PVC). Usually, it does not require any medical treatment. The patients with PAC may experience the feeling of heart stop after a symptom. PVC and PAC are also known as palpitation [7].

\subsection{Neural Networks}

Neural network is the mathematical computing pattern. It models the biological neural system operations. Neural networks have good capability to extract meaningful information from complex and raw data. For this very reason, neural networks can be used to derive models and detect patterns of complex nature which are difficult for human and computer technology. Mostly the multilayer perceptron (MLP) [8], radial basis function (RBF) networks [9], and learning vector quantization (LVQ) networks are used for ECG signal detection and classification. An artificial neural network structure is the inter linkage of many different nonlinear processing elements called neurons. These neurons are connected through weighted synapses to form a network. The function of each neuron is to compute a weighted sum of all synapse inputs, subtract the sum from a predefined bias, and pass the result through a nonlinear sigmoidal (threshold) function whose output ranges between 0 and 1 .

Feedforward backpropagation neural networks (BPNNs) are one of the most common neural network structures, as they are simple and effective. It is used in different kind of machine learning applications such as face recognition etc. The nodes are organized in three layers structure: the input layer, hidden layer and output layer. The input and output layers serve as nodes to buffer input and output for the model, respectively, and the hidden layer serves to provide a means for input relations to be represented in the output [10]. Before any data has been run through the network, the weights for the nodes are random, which has the effect of making the network much like a newborn's brain--developed but without knowledge.

\section{METHODOLOGY}

\subsection{Data Acquisition}

A Vernier ECG Sensor is used which is a 3-lead sensor. It provides electrical isolation with a transformer. It amplifies the body's potentials from $1 \mathrm{mV}$ to $1 \mathrm{~V}$. However the output of the sensor is sufficient for the classification of different diseases and its medications. The data acquisition system can utilize analog or digital filtering techniques or a combination of the two. Analog filters are found in almost every electronic circuit. Analog filters reduce the influence of noise in data acquisition systems. With the analog low-pass filter, high frequency noise and interference can be removed from the signal path prior to the analog-to-digital (A/D) conversion. In contrast, a digital filter is used to reduce the in-band frequency noise. Both the analog filter and the digital filter are utilized. Some popular analog filter designs are the Butterworth, Bessel, and Chebyshev. Fourth order Butterworth analog with the cutoff frequency of $180 \mathrm{~Hz}$. The transfer function of a Butterworth filter consists of all poles and no zeros. The frequency behavior has a maximally flat magnitude response in pass-band. The rate of attenuation in transition band is better than Bessel, but not as good as the Chebyshev filter. This filter type has some overshoot in the time domain, but less than the Chebyshev. The ECG signal is converted to digital information to convert the analog input to its equivalent digital number. Conversion time of the free running mode is $110 \mu \mathrm{s}$. This digital information is sent to the Microcontroller (8051) where it outputs the ECG wave's information. RS-232 connector DB-9 is used as the serial data communication equipment. Standard serial interfacing of microcontroller 8051 (TTL) with PC or any RS232C Standard device, requires TTL to RS232 Level converter. A MAX232 is used for this purpose. It provides 2-channel $\mathrm{RS} 232 \mathrm{C}$ port and requires external $10 \mathrm{uF}$ capacitors. The driver requires a single supply of $+5 \mathrm{~V}$.

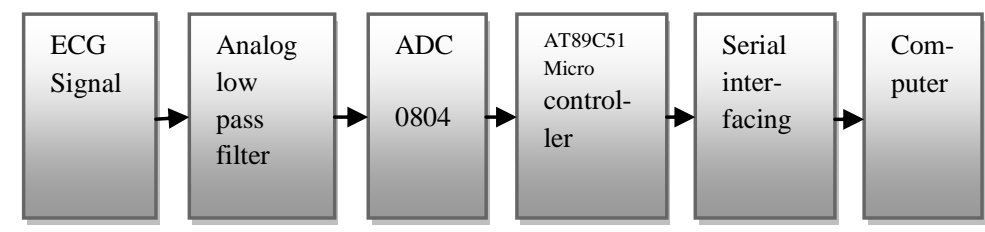

Fig 2: Project Block Diagram

\subsection{Fiducial Points Detection}

In order to classify the ECG signal, the significant features of ECG waveform must be extracted. For ECG parameter or feature extraction the characteristic points of an ECG signal must be determined, i.e., P, Q, R, S, T etc. An algorithm determines these points in the following steps:

first of all the QRS complex is detected by first derivative method.

\section{Signal Slope Calculation}

The slope equation of ECG signal is described by

$$
\begin{gathered}
\text { Slope }(n)=-2 E C G(n-2)-E C G(n-1)+E C G(n+1)+ \\
2 E C G(n+2)
\end{gathered}
$$

Where $\operatorname{ECG}(\mathrm{n})$ represents the amplitude of the nth received discrete ECG signal [11]. 


\section{Slope Threshold Calculation}

slope_threshold $=\max (E C G) \mathrm{x}$ thresh_param $/ 16$

\subsubsection{ECG QRS wave onset Detection}

When two consecutive ECG data satisfy the condition that slope $(n)>$ slope-thresh, the onset of the QRS complex is detected [11]. Normally the waveform of noise interference is high and sharp. That is, the slope of a single noise point usually exceeds the slope threshold and produces a false detection in the algorithm. However, for two consecutive points, if both of the slopes are larger than the slope threshold, these two points are most probably not the noise signals and can be used as the onset of QRS wave. [12]

\subsection{2 $Q$ Wave Detection}

A negative wave at the onset of the QRS complex is defined as $\mathrm{Q}$ wave and the valley is defined as $\mathrm{Q}$ point. The algorithm searches the point where the slope becomes greater than the $\mathrm{Q}$ threshold value then it makes a window of 0.01 seconds interval after the $Q$ point and the minimum point in that window is detected as Q point.

\subsection{3 $R$ Wave Detection}

$\mathrm{R}$ point is detected as the maximum point in the 0.05 seconds interval after the Q point.

\subsubsection{S Wave Detection}

The negative deflection following an $\mathrm{R}$ wave is called $\mathrm{S}$ wave. After detecting $\mathrm{R}$ point, $\mathrm{S}$ point is searched based on duration and zero crossing of the slope [13]

\subsubsection{T Wave Detection}

The moving average filter is applied to smooth the rest of the signal. After the QRS complex has been searched, the T wave is detected by the algorithm based on the threshold value and a positive slop followed by the negative one

\subsubsection{P Wave Detection}

The last wave detected is the P-wave. The P-wave is located between the end of the T-wave and the beginning of a new QRS complex (see Fig 3). The detection rule for a P-wave is the same as for T-wave. The $\mathrm{P}$ wave offset is located by forward search in ECG data, starting from the P point and onset is located by backward search in ECG data starting from the $\mathrm{P}$ point.

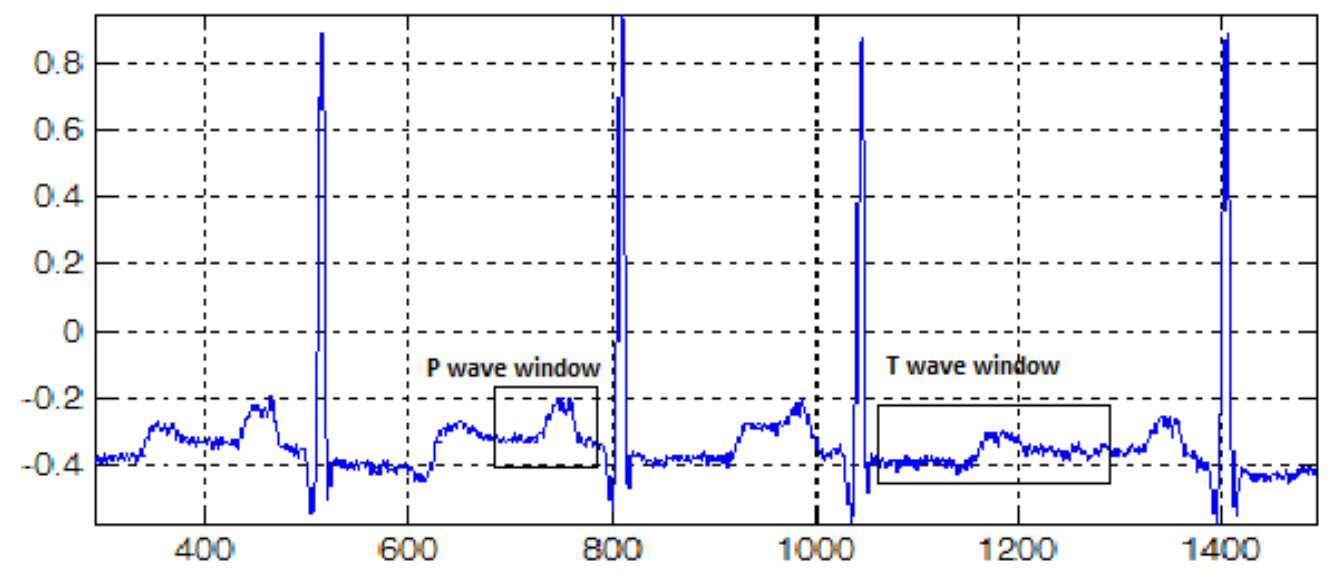

Fig 3: Matlab Image of a real time ECG : Search windows for T- and P-wave location

\subsection{Parameter Extraction}

In automatic heart rhythm classification, the extraction of various parameters or features is of prime importance. A set of important morphological parameters can be extracted after identifying fiducial points. These selected parameters are given as an input to the neural network for classification so that a reasonable classification rate is achieved through small set of features. The following eighteen parameters are evaluated from the fiducial points:

\subsubsection{Time Domain Features}

All these intervals can be calculated by finding the difference between the samples numbers associated with the corresponding points.

\subsubsection{QRS Complex Duration}

Another important feature is QRS complex interval which is used to analyze and classify the ECG signal. This feature is defined as the time taken for depolarization of ventricles. The normal functioning time required for depolarization of ven- tricles is $40 \mathrm{~ms}$ to $90 \mathrm{~ms}$. The blocked bundle branches either right or left cause delays in the depolarization process of ventricles. QRS complex duration is $100 \mathrm{~ms}$ in abnormal case. There is an intraventricular conduction delay when the QRS interval is between 100 to $120 \mathrm{~ms}$. If the QRS complex interval duration exceeds $120 \mathrm{~ms}$, it indicates bundle branch block [13]. Two steps should be taken to measure the QRS complex duration, $1^{\text {st }}$ the area under the complex is evaluated and assumed that the shape of QRS complex is of a triangle, the QRS duration is obtained using the following equation: 


\section{$Q R S$ duration $=2 *($ Area under the $Q R S$ complex $) /(R$-wave} amplitude)

There are many other features which can be extracted from the QRS complex. There exists a relation between QRS complex area and $\mathrm{R}$ wave amplitude. It means if $\mathrm{R}$ wave is small then the area under the QRS complex is small. A powerful feature for classification is evaluated by the product of $\mathrm{R}$ wave amplitude and area under QRS complex.

\subsubsection{R-R Interval}

The distance between two adjacent QRS complexes is termed as RR interval [14]. It shows the heart rate. The normal heart rate is between 60 to $100 \mathrm{bpm}$. Sinus bradycardia occurs in case of high heart rate and tachycardia occurs when heart rate is high. The difference between samples number associated with each $\mathrm{R}$ wave is termed as RR interval of consequent beat.

\subsubsection{P-R Interval}

The time lag from the start of atrial depolarization to the start of ventricle depolarization is defined as PR interval. This is another useful parameter which allows the atrial systole to occur. The PR interval is the time required for the sinus atrial node impulse to spread over the atria and through the AV node. The range for normal PR interval is 120 to $200 \mathrm{~ms}$. The accelerated AV conduction occurs if the PR interval is less than $110 \mathrm{~ms}$. If the PR interval duration is greater than $200 \mathrm{~ms}$ then there is a damage or block to the AV conduction. Careful attention should be paid to the $\mathrm{P}$ wave and P-R interval (as shown in figure 4) when assessing ECG rhythm.

\subsubsection{Q-T Interval}

The QT interval will vary depending on the heart rate, age and gender. Prolonged QT-interval may be associated with delayed ventricular repolarization which may cause ventricular tachyarrhythmia's leading to sudden cardiac death. The normal duration for this segment is $0.3-0.34$ seconds.

\subsubsection{P-Q Interval}

The normal PQ interval is between 0.12 and 0.20 seconds.

\subsubsection{S-T Interval}

The ST segment is important in the diagnosis of ventricular ischemia or hypoxia because under those conditions, the ST segment can become either depressed or elevated. Other time domain features include:

P-T Interval

R-S Interval

P-P Interval

R-T Interval

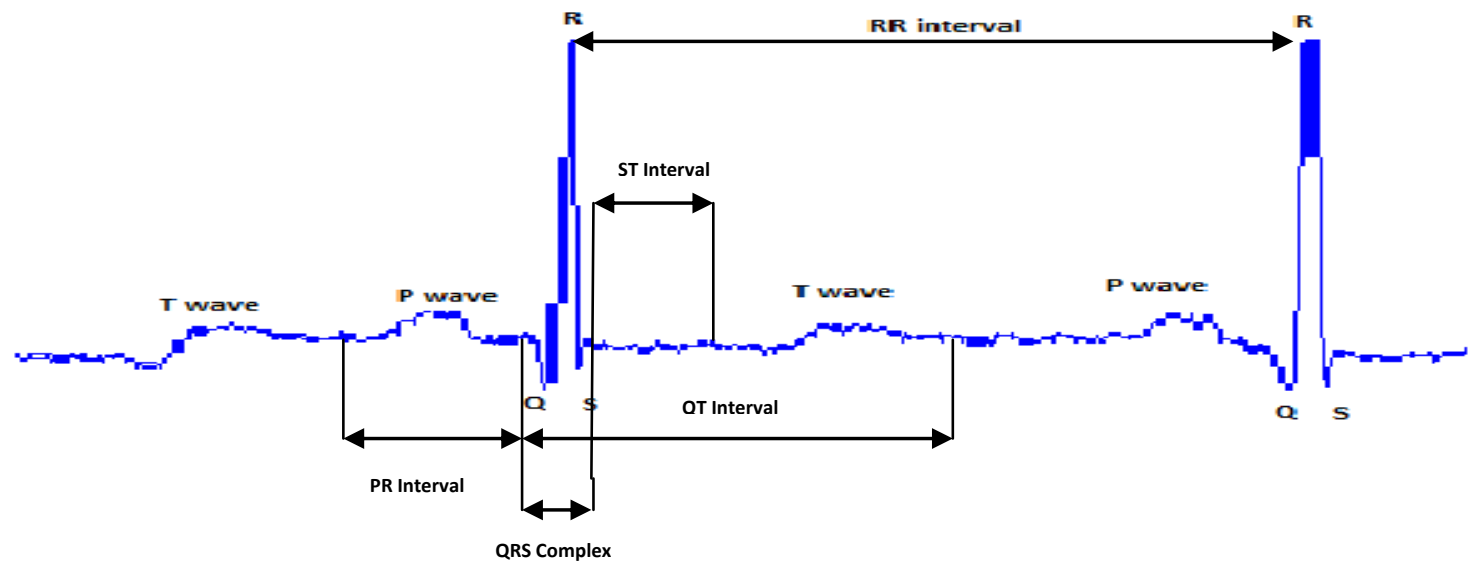

Fig 4: Some Important Features of an ECG

\subsubsection{Amplitude Features}

Significantly elevated or depressed amplitudes away from the baseline are often associated with cardiac illness. Some of the features are calculated for this purpose which is as follows:

\section{Ratio of $R$ amplitude and $Q$ amplitude}

Ratio of $R$ amplitude and $S$ amplitude

Ratio of $P$ amplitude and $Q$ amplitude

Ratio of $T$ amplitude and $S$ amplitude

\subsubsection{Frequency Domain Features}

Frequency domain features provide higher amount of information provided by the signal spectra, compared to time domain methods. The frequency analysis of the surface ECG may be a useful means to augment clinical decision-making [13].
Selected frequency domain features include

\subsubsection{Mean power frequency of the beat}

This feature indicates the distribution of power across the frequency band of $(0-180) \mathrm{Hz}$. The formula for calculating mpf1 is given by

Mpf1 $=\sum_{f(i)=0}^{f(i)=5} f(i) P(f(i)) / \sum_{f(i)=0}^{f(i)=180} P(f(i))$

Where, $P(f(i))$ indicates the power spectral densities (PSD) at frequency $\mathrm{f}(\mathrm{i})$ 


\subsubsection{PSD ratio for the band (0-5) $\mathrm{Hz}$}

Power spectral density indicates the fraction of the power in (0-5) $\mathrm{Hz}$ band.

PSD ratio $=\sum_{f(i)=0}^{f(i)=5} P(f(i)) / \sum_{f(i)=0}^{f(i)=180} P(f(i))$

\subsubsection{PSD ratio for the band (5-10) $\mathrm{Hz}$ for the beat} Indicates fraction of the power in $(5-10) \mathrm{Hz}$ band and is calculated similarly as in (a).

\subsubsection{PSD ratio for the band (10-25) $\mathrm{Hz}$ for the beat} Indicates the fraction of the power in (10-25) Hz band and is calculated similarly as (a). The various types of heart beats and code for their identification are extracted from [15].

Table 2: Arrhythmic Conditions Dealt in our Research

\begin{tabular}{|lll|}
\hline NORMAL & 1 & normal beat \\
\hline LBBB & 2 & left bundle branch block beat \\
\hline RBBB & 3 & right bundle branch block beat \\
\hline PVC & 5 & premature ventricular contraction \\
\hline APC & 8 & atrial premature contraction \\
\hline PACE & 12 & paced beat \\
\hline RHYTHM & 28 & rhythm change \\
\hline
\end{tabular}

\subsection{Neural Network Classifier for Arrhythmia Diagnosis}

After much experimentation with different neural network architectures feed forward backpropagation (multi layer perceptron (MLP)) with three layers is chosen. The input layer consists of 18 neurons corresponding to the extracted features of ECG, the hidden layer consists of 10 neurons and the output layer contains 7 neurons. The neurons in the hidden layer can be increased depending upon the complexity of network [16]. Before training a feed forward network, you must initialize the weights and biases. The activation function of $2^{\text {nd }}$ layer is purelin and of $3^{\text {rd }}$ layer is logsig. These functions are often called "squashing" functions, because they compress an infinite input range into a finite output range. Sigmoid functions are characterized by the fact that their slopes must approach zero as the input gets large.

First of all the peaks detection algorithm is run over all the selected ECG records and detected the set of peaks $(\mathrm{P}, \mathrm{Q}, \mathrm{R}$, $\mathrm{S}, \mathrm{T})$. The program of feature generation is run to assemble all the input features to the network and by making use of annotation data the arrhythmia type for each beat is stored into the target files. All the data is divided into two parts one for training and the other for testing. Neural network training can be made more efficient if certain preprocessing steps are performed on the network inputs and targets. The input data is preprocessed such that it has mean of 0 and a STD deviation of 1 .

Performed principal component analysis to ensure the elements of the input vector set will be uncorrelated also the size of the input vector is reduced by retaining only those components which contribute significantly to the total variation in the data set. The network object is created by defining network training function named Conjugate Backpropagation. Finally the supervised network is trained in Batch mode.
Table 3: Feature extraction results of MIT-BIH databases

\begin{tabular}{|c|c|c|}
\hline No. of records & Arrhythmia Records & Generation \\
\hline 1 & 100 & $\begin{array}{l}\text { Darcantano } \\
99.0765 \%\end{array}$ \\
\hline 2 & 101 & $97.0803 \%$ \\
\hline 3 & 102 & $97.6734 \%$ \\
\hline 4 & 103 & $97.9856 \%$ \\
\hline 5 & 104 & $90.6684 \%$ \\
\hline 6 & 105 & $89.307 \%$ \\
\hline 7 & 106 & $90.5832 \%$ \\
\hline 8 & 107 & $92.4555 \%$ \\
\hline 9 & 108 & $93.035 \%$ \\
\hline 10 & 109 & $98.892 \%$ \\
\hline 11 & 111 & $82.8397 \%$ \\
\hline 12 & 112 & $65.24 \%$ \\
\hline 13 & 114 & $75.7833 \%$ \\
\hline 14 & 115 & $96.6752 \%$ \\
\hline 15 & 116 & $83.3402 \%$ \\
\hline 16 & 117 & $75.0326 \%$ \\
\hline 17 & 118 & $74.5545 \%$ \\
\hline 18 & 119 & $92.0977 \%$ \\
\hline 19 & 121 & $64.5265 \%$ \\
\hline 20 & 122 & $92.1894 \%$ \\
\hline 21 & 123 & $90.1585 \%$ \\
\hline 22 & 124 & $93.9189 \%$ \\
\hline 23 & 200 & $83.9741 \%$ \\
\hline 24 & 202 & $80.7297 \%$ \\
\hline 25 & 203 & $69.4839 \%$ \\
\hline 26 & 205 & $97.4474 \%$ \\
\hline 27 & 207 & $74.9053 \%$ \\
\hline 28 & 208 & $79.0168 \%$ \\
\hline 29 & 209 & $86.6294 \%$ \\
\hline 30 & 210 & $93.4628 \%$ \\
\hline 31 & 212 & $95.0635 \%$ \\
\hline 32 & 213 & $88.2156 \%$ \\
\hline 33 & 214 & $69.4839 \%$ \\
\hline 34 & 215 & $93.8643 \%$ \\
\hline 35 & 217 & $85.1991 \%$ \\
\hline 36 & 219 & $71.91 \%$ \\
\hline 37 & 220 & $96.7507 \%$ \\
\hline 38 & 221 & $95.3971 \%$ \\
\hline 39 & 222 & $77.7524 \%$ \\
\hline 40 & 223 & $94.611 \%$ \\
\hline 41 & 228 & $85.1991 \%$ \\
\hline 42 & 230 & $87.0981 \%$ \\
\hline 43 & 231 & $74.11 \%$ \\
\hline 44 & 233 & $66.6454 \%$ \\
\hline 45 & 234 & $98.4035 \%$ \\
\hline \multicolumn{2}{|c|}{ Average percentage } & $87.2624 \%$ \\
\hline
\end{tabular}


The number of neurons in hidden layer is more difficult to define in a systematic way. Generally, a hidden layer with a relatively large number of nodes tends to produce a more flexible diagnostic model. The output layer composes of 3 nodes and each node corresponds to existence of heart disease. The mean square error (MSE) is 0.012529 with 10000 training iterations. The goal $(0.001)$ is approximately achieved.

\section{RESULS \& DISCUSSIONS \\ 3.1 Feature Generation from ECG Records}

The peaks detection algorithm and features generation program is run over the selected records to assemble the training data.

- Peaks detection algorithm gives the peaks P, Q, R, $\mathrm{S}, \mathrm{T}$.

- The feature generation program gives the 18 frequency and time domain features of ECG samples.

- The extracted rate of all the records is shown in the table 3.

\subsection{Network Simulation for Cardiac Arrhythmia}

For training the network, MIT-BIH databases [17] are used. Total of 100 sample databases are used half for training and half of them for testing the network. The simulation results for test data are shown in table 4 . The test result of a sample ECG signal is shown in figure 5 .

Table 4: Network Simulation Results of Arrhythmic condition

\begin{tabular}{|c|c|}
\hline \multicolumn{2}{|c|}{ NETWORK SIMULATION RESULTS } \\
\hline Correct arrhythmia diagnosis & $78 \%$ \\
\hline Incorrect arrhythmia diagnosis & $22 \%$ \\
\hline Mean Squared Error & 0.0125 \\
\hline
\end{tabular}



Fig 5: Matlab: Test Result of sample ECG signal

\section{CONCLUSION}

There are various methods for peaks detection of ECG waveform for example Neural Network, Wavelet Transform and Template matching etc. but the method based on first derivative is easy to implement and gives good results even though the ECG signal was corrupted by high level of composite noise. The algorithm detects most of the fiducial points successfully although there is some deficiency in $\mathrm{P}$ and $\mathrm{T}$ wave detection. We added two additional cascaded FIR filters to deal with the real time signal in software. The efficiency of the neural network for arrhythmia diagnosis is $78 \%$. It detects arrhythmia disorders accurately. The goal for training is ap- proximately met. The mean squared error is 0.0125 . The network works successfully for real time database.

\section{ACKNOWLEDGMENTS}

We would like to express our gratitude to our Professor Dr. Zubair Ahmed Khan for his support and assistance in guiding us throughout. We are grateful to the Department of Electrical Engineering for making this research work possible. Thanks to our family members for their continuous help and support.

\section{REFERENCES}

[1] Denis Berdajs, Marko Turina, 2010, "Operative Anatomy of the Heart".

[2] The Standard 12 Lead ECG: http://library.med.utah.edu/kw/ecg/ecg_outline/Lesson $1 / \mathrm{i}$ ndex.html

[3] Basic ECG Interpretation Tutorial: http://www.mauvila.com/ECG/ecg_intro.htm

[4] ECG: http"//www.mauvila.com/ECG/ecg_intro.htm

[5] Tom Kenny, 2011, "The Nuts and Bolts of Cardiac Pacing".

[6] Samuel Bellet, 1971, "Clinical disorders of the heart beat".

[7] Fawzy G. Estafanous, Paul G. Barash, J. G. Reves, 1994, "Cardiac anesthesia: principles and clinical practice".

[8] R. Acharya, P.S. Bhat, S.S. Iyengar, A. Roo and S. Dua, 2003. Classification of heart rate data using artificial neural network and fuzzy equivalence relation, Pattern Recognition, vol. 36, no. 1, pp. 61-68.

[9] Y. Ozbay and B. Karlik, 2001. A recognition of ECG arrhythmias using artificial neural network, Proceedings of the 23rd Annual Conference, IEEE/EMBS, Istanbul, Turkey, 2001.

[10] D.H. Wolpert, 1992. Stacked generalization, Neural Networks, vol. 5, pp. 241-259.

[11] K. F. Tan, K. L. Chan, and K. Choi, 2000. Detection of the QRS Complex, P Wave and T Wave In Electrocardiogram, IEEE Transactions on BME, vol. 45, pp 10771080.

[12] Ren-Guey Lee, I-Chi Chow, Chien-Chih Lai, Ming-Hsiu Liu, Min-Jang Chiu, 2005. A Novel QRS Detection Algorithm Applied to the Analysis for Heart Rate Variability of Patients with Sleep Apnea, IEEE Transactions on BME, vol. 17, no.5, pp 258-262.

[13] H.Gholam-Hosseini and H.Nazeran, 1998. Detection and Extraction of the ECG Signal Parameters, IEEE Transactions on BME, vol. 20, no.1, pp. 127-130.

[14] T Srikantha, SA Napperb, H Guc, 2002. A Bottom-Up Design of Neural Network Based ECG Beat and Rulebased Rhythm Classifier, IJBEM, vol. 4, No. 1.

[15] Codes for beat identification: http://www.physionet.org/

[16] S N Sivanandam, S Sumathi, S N Deepa, "Introduction to Neural Networks using Matlab: a computer engineering series", McGraw-Hill.

[17] R.G. Mark and G.B. Moody, MIT-BIH Arrhythmia Database $1997 . \quad$ Available from: http://www.ecg.mit.edu/dbinfo.html. 